\title{
HAUSDORFF DIMENSION OF A SET IN THE THEORY OF CONTINUED FRACTIONS
}

\author{
AYREENA BAKHTAWAR, PHILIP BOS, AND MUMTAZ HUSSAIN
}

ABStract. In this article we calculate the Hausdorff dimension of the set

$$
\mathcal{F}(\Phi)=\left\{x \in[0,1): \begin{array}{rl}
a_{n+1}(x) a_{n}(x) & \geq \Phi(n) \text { for infinitely many } n \in \mathbb{N} \text { and } \\
a_{n+1}(x) & <\Phi(n) \text { for all sufficiently large } n \in \mathbb{N}
\end{array}\right\}
$$

where $\Phi: \mathbb{N} \rightarrow(1, \infty)$ is any function with $\lim _{n \rightarrow \infty} \Phi(n)=\infty$. This in turn contributes to the metrical theory of continued fractions as well as gives insights about the set of Dirichlet non-improvable numbers.

\section{INTRODUCTION}

Metric Diophantine approximation is concerned with the quantitative analysis of the density of rationals in the reals. We commence with the famous uniform Diophantine approximation result, Dirichlet's theorem, which is a simple consequence of the pigeon-hole principle.

Theorem 1.1 (Dirichlet 1842). Given $x \in \mathbb{R}$ and $t>1$, there exists integers $p$, $q$ such that

$$
|q x-p| \leq \frac{1}{t} \quad \text { and } \quad 1 \leq q<t .
$$

The above result is uniform in the sense that it ensures non-trivial integer solution for all $t$. It can be easily seen that the rate of approximation given in (1.1) improves the trivial rate of $1 / 2$. A natural question that arises here is, what happens if the right hand side of (1.1) is replaced with a faster decreasing function depending upon $t$ ? To this end, let $\psi:\left[t_{0}, \infty\right) \rightarrow \mathbb{R}^{+}$ be any monotonically decreasing function, where $t_{0} \geq 1$ is fixed. Denote by $D(\psi)$ the set all those real numbers $x$ for which the system

$$
|q x-p| \leq \psi(t) \text { and }|q|<t
$$

guarantees a nontrivial integer solution for all large enough $t$. A real number $x \in D(\psi)$ (resp. $\left.x \in D(\psi)^{c}\right)$ will be referred to as an $\psi$-Dirichlet improvable (resp. $\psi$-Dirichlet non-improvable) number.

Davenport-Schmidt [4] proved that the set $D(k / t)$ has a Lebesgue measure zero for any $k<1$ by showing that $D(k / t)$ is a subset of the union of the set of rationals $\mathbb{Q}$ and the set of badly approximable numbers. The set $D(\psi)$ is connected with the continued fractions as observed by Kleinbock-Wadleigh [11, Lemma 2.2] proving that an irrational number is $\psi$ Dirichlet improvable if and only if the product of consecutive partial quotients of the continued fraction expansion of that number do not grow fast. To state their result as well as our main result, first we introduce some necessary definitions and notations.

Every irrational $x \in[0,1)$ can be uniquely expressed as a simple infinite continued fraction expansion of the form:

$$
x=\left[a_{1}(x), a_{2}(x), \ldots,\right],
$$

The research of A. Bakhtawar is supported by La Trobe University postgraduate research award.

The research of M. Hussain is supported by La Trobe University startup grant. 
where $a_{n}(x) \in \mathbb{N}, n \geq 1$ are known as the partial quotients of $x$. This expansion can be induced by the Gauss map $T:[0,1) \rightarrow[0,1)$ defined as

$$
T(0):=0, \quad T(x):=\frac{1}{x}(\bmod 1), \quad \text { for } x \in(0,1),
$$

with $a_{1}(x)=\left\lfloor\frac{1}{x}\right\rfloor$, where $\lfloor$.$\rfloor represents the floor function and a_{n}(x)=a_{1}\left(T^{n-1}(x)\right)$ for $n \geq 2$.

The metrical theory of continued fractions which focuses on investigating the properties of partial quotients for almost all $x \in[0,1)$ is one of the important areas of research in the study of continued fractions and is closely connected with the Diophantine approximation. The main connection is that the convergents of a real number $x$ are good rational approximates for $x$. In fact, for any $\tau>0$ the famous Jarník-Besicovitch set

$$
\left\{x \in[0,1):\left|x-\frac{p}{q}\right|<\frac{1}{q^{\tau+2}} \text { for infinitely many }(p, q) \in \mathbb{Z} \times \mathbb{N}\right\},
$$

is equivalent to the following set,

$$
\left\{x \in[0,1): a_{n}(x) \geq q_{n}^{\tau}(x) \text { for infinitely many } n \in \mathbb{N}\right\} .
$$

For further details about this connection we refer to [6]. Thus a real number $x$ is $\tau$-approximable if the partial quotients in its continued fraction expansion are growing fast. Therefore the growth rate of the partial quotients reveals how well a real number can be approximated by rationals.

A starting point in the metrical theory of continued fractions is the well-known BorelBernstein theorem [2, 3] which gives an analogue of Borel-Cantelli '0-1' law with respect to Lebesgue measure for the set of real numbers with large partial quotients. A lot of work has been done in the direction of improving Borel-Bernstein's theorem, for example, estimation of Hausdorff dimension of sets when partial quotients $a_{n}(x)$ obeys different conditions has been studied in [5, 6, 13].

Throughout this paper, let $\Phi: \mathbb{N} \rightarrow(1, \infty)$ be an arbitrary function such that $\lim _{n \rightarrow \infty} \Phi(n)=\infty$,

$$
\mathcal{E}_{1}(\Phi):=\left\{x \in[0,1): a_{n}(x) \geq \Phi(n) \text { for infinitely many } n \in \mathbb{N}\right\} .
$$

Theorem 1.2 ([3, Borel-Bernstein]). The Lebesgue measure of $\mathcal{E}_{1}(\Phi)$ is is either zero or full according as the series $\sum_{n=1}^{\infty} 1 / \Phi(n)$ converges or diverges respectively.

The Borel-Bernstein's theorem is a remarkably simple dichotomy result but it fails to distinguish between Lebesgue null sets, that is, it gives Lebesgue measure zero for sets $\mathcal{E}_{1}(\Phi)$ for rapidly increasing functions $\Phi$. To distinguish between Lebesgue null sets the notion of Hausdorff measure and dimension are the appropriate tools and has gained much importance in the metrical theory of continued fractions. Keeping this in view Wang-Wu [19] completely determined the Hausdorff dimension of the set $\mathcal{E}_{1}(\Phi)$.

Theorem $1.3\left(\left[19\right.\right.$, Wang-Wu]). Let $\Phi: \mathbb{N} \rightarrow \mathbb{R}^{+}$be an arbitrary positive function. Suppose

$$
\log B=\liminf _{n \rightarrow \infty} \frac{\log \Phi(n)}{n} \text { and } \log b=\liminf _{n \rightarrow \infty} \frac{\log \log \Phi(n)}{n} .
$$

(i) When $B=1$, $\operatorname{dim}_{\mathrm{H}} \mathcal{E}_{1}(\Phi)=1$.

(ii) When $B=\infty$, $\operatorname{dim}_{\mathrm{H}} \mathcal{E}_{1}(\Phi)=1 /(1+b)$.

(iii) When $1<B<\infty, \operatorname{dim}_{\mathrm{H}} \mathcal{E}_{1}(\Phi)=s_{B}=\inf \left\{s \geq 0: \mathrm{P}\left(T,-s\left(\log B+\log \left|T^{\prime}\right|\right)\right) \leq 0\right\}$,

where $T$ is the Gauss map related to the continued fraction expansion, $T^{\prime}$ denotes the derivative of Tand $\mathrm{P}$ represents the pressure function defined in Section 3 .

The following result illustrates the continuity of dimensional number $s_{B}$ and shows that its limit exist. 
Proposition $1.4\left(\left[19\right.\right.$, Wang-Wu]). The parameter $s_{B}$ is continuous with respect to $B$, and

$$
\lim _{B \rightarrow 1} s_{B}=1, \lim _{B \rightarrow \infty} s_{B}=1 / 2 .
$$

The set $\mathcal{E}_{1}(\Phi)$ is connected with the Jarnik-Besicovitch set (1.3) in the sense that in (1.3) the approximating function depends on the $n$th convergent of $x$ " $q_{n}(x)$ " whereas in $\mathcal{E}_{1}(\Phi)$ the approximating function $\Phi$ is a function of index " $n$ ". Recall that $\mathcal{E}_{1}(\Phi)$ consist of real numbers such that one partial quotient grows very fast but as we move towards the product of two consecutive partial quotients, the corresponding set of real numbers is linked with the set of Dirichlet non-improvable numbers (as observed by Kleinbock-Wadleigh [11]) in the following sense.

There exists $w>1$ such that for every $x \notin \mathbb{Q}, w^{n} \leq q_{n}(x)$ for all $n \geq 2$. There also exits $W>w$ such that for almost every $x, q_{n}(x) \leq W^{n}$ for all large enough $n$ (see [10, §14]).

This leads to the following criteria for Dirichlet improvability.

Lemma 1.5 ([11, Kleinbock-Wadleigh]). Let $x \in[0,1) \backslash \mathbb{Q}$, and let $\psi:\left[t_{0}, \infty\right) \rightarrow \mathbb{R}^{+}$be non-increasing function with $t \psi(t)<1$ for all $t \geq t_{0}$ and $\Phi(t)=\frac{t \psi(t)}{1-t \psi(t)}$. Then

(i) $x \in D(\psi)$ if $a_{n+1}(x) a_{n}(x) \leq \Phi\left(w^{n}\right) / 4$ for all sufficiently large $n$.

(ii) $x \in D(\psi)^{c}$ if $a_{n+1}(x) a_{n}(x)>\Phi\left(W^{n}\right)$ for infinitely many $n$.

Thus this lemma characterizes a real number $x$ to be $\psi$-Dirichlet non-improvable in terms of the growth of product of consecutive partial quotients. Further, Kleinbock-Wadleigh also proved a zero-one law for the Lebesgue measure of $D(\psi)$. With a change of notation, we consider the set

$$
\mathcal{E}_{2}(\Phi):=\left\{x \in[0,1): a_{n}(x) a_{n+1}(x) \geq \Phi(n) \text { for infinitely many } n \in \mathbb{N}\right\},
$$

where $\Phi: \mathbb{N} \rightarrow(1, \infty)$ is any function with $\lim _{n \rightarrow \infty} \Phi(n)=\infty$.

Theorem 1.6 ([11, Kleinbock-Wadleigh]). The Lebesgue measure of $\mathcal{E}_{2}(\Phi)$ is is either zero or full according as the series $\sum_{n=1}^{\infty} \frac{\log \Phi(n)}{\Phi(n)}$ converges or diverges respectively.

Note that the $\mathcal{E}_{1}(\Phi)$ is properly contained in $\mathcal{E}_{2}(\Phi)$. Since the inclusion is proper, this raises a natural question of the size of the set $\mathcal{E}_{2}(\Phi) \backslash \mathcal{E}_{1}(\Phi)$. In other words, a natural question is to estimate the size of the set

$\mathcal{F}(\Phi):=\mathcal{E}_{2}(\Phi) \backslash \mathcal{E}_{1}(\Phi)=\left\{x \in[0,1): \begin{array}{rl}a_{n+1}(x) a_{n}(x) & \geq \Phi(n) \text { for infinitely many } n \in \mathbb{N} \text { and } \\ a_{n+1}(x) & <\Phi(n) \text { for all sufficiently large } n \in \mathbb{N}\end{array}\right\}$,

in terms of Hausdorff dimension. Note that the set $\mathcal{F}(\Phi)$ arises by excluding the set of well approximable points (c.f. Jarník-Besicovitch set (1.3) ) from the set $\mathcal{E}_{2}(\Phi)$ of Dirichlet nonimprovable points expressed in terms of their continued fraction entries. We prove that the set $\mathcal{F}(\Phi)$ is quite big in a sense that it is uncountable by proving that its Hausdorff dimension is positive.

Theorem 1.7. Let $\Phi: \mathbb{N} \rightarrow(1, \infty)$ be any function with $\lim _{n \rightarrow \infty} \Phi(n)=\infty$. Suppose

$$
\log B=\liminf _{n \rightarrow \infty} \frac{\log \Phi(n)}{n} \text { and } \log b=\liminf _{n \rightarrow \infty} \frac{\log \log \Phi(n)}{n} .
$$

Then

$$
\operatorname{dim}_{\mathrm{H}} \mathcal{F}(\Phi)= \begin{cases}t_{B}=\inf \left\{s \geq 0: \mathrm{P}\left(T,-s^{2} \log B-s \log \left(\left|T^{\prime}\right|\right) \leq 0\right\}\right. & \text { if } 1<B<\infty \\ \frac{1}{1+b} & \text { if } B=\infty,\end{cases}
$$

where $\mathrm{P}$ represents the pressure function defined in Section 3 . 
Note that if we take $B=1$ then from the definition of $\mathcal{F}(\Phi)$ we have $a_{n+1}(x)<1$ which is a contradiction to the assumption that $a_{n+1}(x) \geq 1$. Therefore, $B$ is strictly greater than 1 .

When $\Phi$ is a function of the $n$th convergents $\left(q_{n}(x)\right)$ then the Hausdorff dimension of the set $\mathcal{F}(\Phi)$ has been established by the authors in [1], and the Hausdorff measure theoretic results for the set $\mathcal{E}_{2}(\Phi)$ have been established by Hussain-Kleinbock-Wadleigh-Wang in [9]. The Hausdorff dimension of level sets within this setup are investigated very recently by Huang-Wu [8].

\section{Preliminaries}

In this section we aim to gather some fundamental properties of continued fractions, pressure function and a few auxiliary results that will be helpful for obtaining the Hausdorff dimension of $\mathcal{F}(\Phi)$.

2.1. Continued fractions and Diophantine approximation. For any vector $\left(a_{1}, \ldots, a_{n}\right) \in \mathbb{N}^{n}$ with any $n \in \mathbb{N}$, we call

$$
I_{n}\left(a_{1}, a_{2}, \ldots, a_{n}\right)= \begin{cases}{\left[\frac{p_{n}}{q_{n}}, \frac{p_{n}+p_{n-1}}{q_{n}+q_{n-1}}\right)} & \text { if } n \text { is even; } \\ \left(\frac{p_{n}+p_{n-1}}{q_{n}+q_{n-1}}, \frac{p_{n}}{q_{n}}\right] & \text { if } n \text { is odd, }\end{cases}
$$

a "basic cylinder" of order $n$, where $p_{n}, q_{n}$ are generated by the following recursive relation

$$
\begin{aligned}
& p_{-1}=1, p_{0}=0, p_{n+1}=a_{n+1} p_{n}+p_{n-1}, \\
& q_{-1}=0, q_{0}=1, q_{n+1}=a_{n+1} q_{n}+q_{n-1} .
\end{aligned}
$$

In fact the basic cylinder of order $n$ represents the set of those real numbers in $[0,1)$ that have continued fraction expansion starting from $a_{1}, \ldots, a_{n}$, that is

$$
I_{n}=I_{n}\left(a_{1}, \ldots, a_{n}\right):=\left\{x \in[0,1): a_{1}(x)=a_{1}, \ldots, a_{n}(x)=a_{n}\right\} .
$$

From [10] it is well known that the length of $I_{n}$ is

$$
\frac{1}{2 q_{n}^{2}} \leq\left|I_{n}\left(a_{1}, \ldots, a_{n}\right)\right|=\frac{1}{q_{n}\left(q_{n}+q_{n-1}\right)} \leq \frac{1}{q_{n}^{2}},
$$

since $p_{n-1} q_{n}-p_{n} q_{n-1}=(-1)^{n}$, for all $n \geq 1$.

For any $n \geq 1$ and irrational $x \in[0,1)$, let $p_{n}(x)=p_{n}$ and $q_{n}(x)=q_{n}$ be given by 2.2 , define $\frac{p_{n}(x)}{q_{n}(x)}$ "the $n$th convergent of $x$ " by

$$
\frac{p_{n}(x)}{q_{n}(x)}:=\left[a_{1}(x), \ldots, a_{n}(x)\right] \quad(n \geq 1)
$$

From (2.2) note that for any $n \geq 1, q_{n}$ is determined by $a_{1}, \ldots, a_{n}$. Therefore, we can write $q_{n}=q_{n}\left(a_{1}, \ldots, a_{n}\right)$. Just to avoid confusion we can use $a_{n}$ and $q_{n}$ in place of $a_{n}(x)$ and $q_{n}(x)$, respectively.

Proposition 2.1 ([10, Khintchine]). Let $k \geq 1, n \geq 1$ and $a_{1}, \ldots, a_{n}$ be positive integers. Then we have

$\left(\mathrm{P}_{1}\right) q_{n} \geq 2^{(n-1) / 2}$ and for any $1 \leq k \leq n$,

$$
\frac{a_{k}+1}{2} \leq \frac{q_{n}\left(a_{1}, \ldots, a_{n}\right)}{q_{n}\left(a_{1}, \ldots, a_{k-1}, a_{k+1} \ldots, a_{n}\right)} \leq a_{k}+1
$$

$\left(\mathrm{P}_{2}\right)$

$$
\begin{aligned}
& q_{n+k}\left(a_{1}, \ldots, a_{n}, a_{n+1} \ldots, a_{n+k}\right) \geq q_{n}\left(a_{1}, \ldots, a_{n}\right) q_{k}\left(a_{n+1}, \ldots, a_{n+k}\right), \\
& q_{n+k}\left(a_{1}, \ldots, a_{n}, a_{n+1} \ldots, a_{n+k}\right) \leq 2 q_{n}\left(a_{1}, \ldots, a_{n}\right) q_{k}\left(a_{n+1}, \ldots, a_{n+k}\right) .
\end{aligned}
$$


$\left(\mathrm{P}_{3}\right)$

$$
\frac{1}{3 a_{n+1} q_{n}^{2}}<\left|x-\frac{p_{n}}{q_{n}}\right|=\frac{1}{q_{n}\left(q_{n+1}+T^{n+1} x q_{n}\right)}<\frac{1}{a_{n+1} q_{n}^{2}},
$$

and for any $n \geq 1$ the derivative of $T^{n}$ is given by

$$
\left(T^{n}\right)^{\prime}(x)=\frac{(-1)^{n}}{\left(x q_{n-1}-p_{n-1}\right)^{2}} .
$$

The next theorem known as Legendre's Theorem, connects 1-dimensional Diophantine approximation with continued fractions.

Theorem 2.2 (Legendre). Let $\frac{p}{q}$ be a rational number. Then

$$
\left|x-\frac{p}{q}\right|<\frac{1}{2 q^{2}} \Longrightarrow \frac{p}{q}=\frac{p_{n}(x)}{q_{n}(x)}, \quad \text { for some } n \geq 1 \text {. }
$$

According to Legendre's theorem if an irrational $x$ is well approximated by a rational $\frac{p}{q}$, then this rational must be a convergent of $x$. Thus in order to find good rational approximates to an irrational number we only need to focus on its convergents. Note that, from $\left(\mathrm{P}_{3}\right)$ of Proposition 2.1, a real number $x$ is well approximated by its convergent $\frac{p_{n}}{q_{n}}$ if its $(n+1)$ th partial quotient $\left(a_{n+1}\right)$ is sufficiently large.

The next result is due to Łuczak [13].

Lemma 2.3 ([13, Luczak $])$. For any $a, b>1$, the sets

$$
\left\{x \in[0,1): a_{n}(x) \geq a^{b^{n}}, \text { for infinitely many } n \in \mathbb{N}\right\}
$$

and

$$
\left\{x \in[0,1): a_{n}(x) \geq a^{b^{n}}, \text { for all sufficiently large } n \in \mathbb{N}\right\}
$$

are of the same Hausdorff dimension $\frac{1}{1+b}$.

\section{Pressure function and Hausdorff dimension}

We collect some basic details about the so-called "pressure function" and its connection with infinite systems generated by continued fractions.

Walters [18] explains the concept of topological pressure and the pressure function in general. For our purposes, we seek to utilise key concepts that are specialised to the continued fraction setting. Guiding the reader through the references, the end game is to produce a function, from which we can produce a lower bound for the Hausdorff dimension of our set of interest.

Let $T: X \rightarrow X$ be a continuous transformation of a compact metric space $(X, d)$. Let $C(X, \mathbb{R})$ denote the Banach algebra of real-valued continuous functions of $X$ equipped with the supremum norm. The topological pressure of $T$ will be a map $\mathrm{P}(T, \cdot): C(X, \mathbb{R}) \rightarrow \mathbb{R} \cup \infty$.

In the case of many linear maps, which includes self similar sets, the dimension can be found implicitly in terms of an expression involving only the rates of contraction. A theorem of Moran (1946) can then be used to calculate the Hausdorff dimension of self-similar sets (see Theorem 2.2.1 of [17]). In the non-linear case, however, the corresponding generalisation of Moran, involves the so called pressure function.

Definition 3.1. Given any continuous function $f: X \rightarrow \mathbb{R}$ we define its pressure $\mathbf{P}(f)$ (with respect to $T$ ) as

$$
\mathrm{P}(f):=\limsup _{n \rightarrow \infty} \frac{1}{n} \log \underbrace{\left(\sum_{\substack{T^{n} x=x \\ x \in \bar{X}}} e^{f(x)+f(T x)+\cdots+f\left(T^{n-1} x\right)}\right)}_{\text {Sum over periodic points }}
$$


As is seen in [17, the limit actually exists and so the "lim sup" can actually be replaced by a "lim". In practice, we shall mainly be interested in a family of functions $f_{t}(x)=-t \log \left|T^{\prime}(x)\right|$, $x \in X$ and $0 \leq t \leq d$, so that the above function reduces to

$$
[0, d] \rightarrow \mathbb{R}, \quad t \mapsto \mathrm{P}\left(f_{t}\right)=\limsup _{n \rightarrow \infty} \frac{1}{n} \log \left(\sum_{\substack{T^{n} x=x \\ x \in X}} \frac{1}{\left|\left(T^{n}\right)^{\prime}(x)\right|^{t}}\right)
$$

The following standard result is essentially due to Bowen and Ruelle. Bowen showed the result in the context of quasi-circles and Ruelle developed the method for the case of hyperbolic Julia sets.

Theorem 3.2 (Bowen-Ruelle). Let $T: X \rightarrow X$ be a $C^{1+\alpha}$ conformal expanding map. There is a unique solution $0 \leq s \leq d$ to

$$
\mathrm{P}\left(-s \log \left|T^{\prime}\right|\right)=0
$$

which occurs precisely at $s=\operatorname{dim}_{\mathrm{H}} X$.

Proof. See Theorem 2.3.2 of [17].

Finally, we observe that the function $t \mapsto \mathbf{P}\left(f_{t}\right)$ has the following interesting properties:

(i) $\mathrm{P}(0)=\log k$;

(ii) $t \mapsto \mathbf{P}\left(f_{t}\right)$ is strictly monotone decreasing; and

(iii) $t \mapsto \mathrm{P}\left(f_{t}\right)$ is analytic on $[0, d]$.

Property (i) is immediate from the definition. For the proofs of properties (ii) and (iii) see page 32 of [17].

The above ideas are sufficient background to explain the reason for the use of pressure functions in Hausdorff dimension calculations for fractal sets of continued fractions.

The Continued Fraction Setting: For more thorough results on pressure function in infinite conformal iterated function systems, we refer to [14, 15, 16]. After defining the limit set, they prove an analogue of the Moran-Bowen formula, identifying its Hausdorff dimension as the zero of the pressure function $\mathrm{P}(t)$. Mauldin-Urbański [15] presented a form of pressure function in conformal iterated function systems with applications to the geometry of continued fractions.

From these papers, a pressure function with a continuous potential can be approximated by the pressure function restricted to the subsystems in continued fraction.

Let us consider a finite or infinite subset $\mathcal{A}$ of $\mathbb{N}$ and define

$$
Y_{\mathcal{A}}=\left\{x \in[0,1) \text { : for all } n \geq 1, a_{n}(x) \in \mathcal{A}\right\} .
$$

Then $\left(Y_{\mathcal{A}}, T\right)$ is a subsystem of $([0,1), T)$ where $T$ is a Gauss map as defined in equation (1.2). Given any real function $\varphi:[0,1) \rightarrow \mathbb{R}$, the pressure function restricted to the system $\left(Y_{\mathcal{A}}, T\right)$ is defined as

$$
\mathrm{P}_{\mathcal{A}}(T, \varphi):=\lim _{n \rightarrow \infty} \frac{1}{n} \log \sum_{a_{1}, \cdots, a_{n} \in \mathcal{A}} \sup _{x \in Y_{\mathcal{A}}} e^{S_{n} \varphi\left(\left[a_{1}, \cdots, a_{n}+x\right]\right)},
$$

where $S_{n} \varphi(x)$ denotes the ergodic sum $\varphi(x)+\cdots+\varphi\left(T^{n-1} x\right)$. Denote $\mathrm{P}_{\mathbb{N}}(T, \varphi)$ by $\mathrm{P}(T, \varphi)$ for $\mathcal{A}=\mathbb{N}$. Also note that if $\varphi$ satisfy the continuity property than we can remove the supremum from equation (3.1).

For each $n \geq 1$ we represent the $n$th variation of $\varphi$ by

$$
\operatorname{Var}_{n}(\varphi):=\sup \left\{|\varphi(x)-\varphi(y)|: I_{n}(x)=I_{n}(y)\right\}
$$

The existence of the limit in equation (3.1) is due to the following result. 
Proposition $3.3\left(\left[12\right.\right.$, Proposition 2.4]). Let $\varphi:[0,1) \rightarrow \mathbb{R}$ be a real function with $\operatorname{Var}_{1}(\varphi)<\infty$ and $\operatorname{Var}_{n}(\varphi) \rightarrow 0$ as $n \rightarrow \infty$. Then the limit defining $\mathrm{P}_{\mathcal{A}}(T, \varphi)$ exists and the value of $\mathrm{P}_{\mathcal{A}}(T, \varphi)$ remains the same even without taking supremum over $x \in Y_{\mathcal{A}}$ in (3.1).

The next result by Hanus, Mauldin and Urbański [7] shows that when the system $([0,1), T)$ is approximated by its subsystems $\left(Y_{\mathcal{A}}, T\right)$ then the pressure function has a continuity property in the system of continued fractions (for an elementary proof see [7] or [12]).

Proposition 3.4 ([7, Proposition 2]). Let $\varphi:[0,1) \rightarrow \mathbb{R}$ be a real function with $\operatorname{Var}_{1}(\varphi)<\infty$ and $\operatorname{Var}_{n}(\varphi) \rightarrow 0$ as $n \rightarrow \infty$. We have

$$
\mathrm{P}_{\mathbb{N}}(T, \varphi)=\sup \left\{\mathrm{P}_{\mathcal{A}}(T, \varphi): \mathcal{A} \text { is a finite subset of } \mathbb{N}\right\} .
$$

From now onwards we consider the specific potential

$$
\varphi_{1}(x)=-s\left(s \log B+\log \left|T^{\prime}(x)\right|\right)
$$

where $1<B<\infty, s \geq 0$ and $T^{\prime}$ is the derivative of Gauss map $T$. By applying Proposition 3.4 to $\varphi_{1}$, it is clear that $\varphi_{1}$ satisfies the variation condition.

By using equation 2.7 of Proposition 2.1, it is easy to check that

$$
S_{n}\left(-s\left(s \log B+\log \left|T^{\prime}(x)\right|\right)\right)=-n s^{2} \log B-s \log q_{n}^{2} .
$$

Therefore, the pressure function (3.1) with potential $\varphi_{1}$ becomes

$$
\begin{aligned}
\mathrm{P}_{\mathcal{A}}\left(T, s\left(s \log B+\log \left|T^{\prime}(x)\right|\right)\right) & =\lim _{n \rightarrow \infty} \frac{1}{n} \log \sum_{a_{1}, \ldots, a_{n} \in \mathcal{A}} e^{S_{n}\left(-s\left(s \log B+\log \left|T^{\prime}(x)\right|\right)\right)} \\
& =\lim _{n \rightarrow \infty} \frac{1}{n} \log \sum_{a_{1}, \ldots, a_{n} \in \mathcal{A}}\left(\frac{1}{B^{n s} q_{n}^{2}}\right)^{s} .
\end{aligned}
$$

For any $n \geq 1$ and $s \geq 0$, let

$$
g_{n}(s)=\sum_{a_{1}, \ldots, a_{n} \in \mathcal{A}} \frac{1}{\left(B^{n s} q_{n}^{2}\right)^{s}} .
$$

Define

$$
\begin{gathered}
t_{n, B}(\mathcal{A})=\inf \left\{s \geq 0: g_{n}(s) \leq 1\right\}, \\
t_{B}(\mathcal{A})=\inf \left\{s \geq 0: \mathrm{P}_{\mathcal{A}}\left(T,-s\left(s \log B+\log \left|T^{\prime}\right|\right)\right) \leq 0\right\}, \\
t_{B}(\mathbb{N})=\inf \left\{s \geq 0: \mathrm{P}\left(T,-s\left(s \log B+\log \left|T^{\prime}\right|\right)\right) \leq 0\right\} .
\end{gathered}
$$

If we take $\mathcal{A}$ to be a finite subset of $\mathbb{N}$, then it is easy to check that both $g_{n}(s)$ and $\mathrm{P}_{\mathcal{A}}\left(T,-s\left(s \log B+\log \left|T^{\prime}\right|\right)\right)$ are monotonically decreasing and continuous with respect to $s$ (for details see [19]). Therefore, $t_{n, B}(\mathcal{A})$ and $t_{B}(\mathcal{A})$ are respectively the unique solutions to $g_{n}(s)=1$ and $\mathrm{P}_{\mathcal{A}}\left(T,-s\left(s \log B+\log \left|T^{\prime}\right|\right)\right)=0$.

For any $M \in \mathbb{N}$, take $\mathcal{A}_{M}=\{1,2, \ldots, M\}$. For simplicity, write $t_{n, B}(M)$ for $t_{n, B}\left(\mathcal{A}_{M}\right)$, $t_{B}(M)$ for $t_{B}\left(\mathcal{A}_{M}\right), t_{n, B}$ for $t_{n, B}(\mathbb{N})$ and $t_{B}$ for $t_{B}(\mathbb{N})$.

From Proposition 3.4 and the definition of $t_{n, B}(M)$ we have the following result.

Corollary 3.5. For any integer $M \in \mathbb{N}$,

$$
\lim _{n \rightarrow \infty} t_{n, B}(M)=t_{B}(M), \quad \lim _{M \rightarrow \infty} t_{B}(M)=t_{B} .
$$

Since the function of $B$ belongs to $(1, \infty)$, therefore the dimensional number $t_{B}$ is continuous with respect to $B$ and

$$
\lim _{B \rightarrow 1} t_{B}=1, \quad \lim _{B \rightarrow \infty} t_{B}=1 / 2 .
$$


Proof. This can be proved by following similar steps as for $s_{B}$ in [19].

Also note that from equation 2.4 and definition of $t_{n, B}(M)$, we have $0 \leq t_{B}(M) \leq 1$.

\section{Proof of Theorem 1.7}

Proof. The proof of Theorem 1.7 consist of two cases:

(i) When $1<B<\infty$;

(ii) When $B=\infty$.

\subsection{Case 1. When $1<B<\infty$.}

By the choice of $B$ in the statement of Theorem 1.7 one can easily note that

$$
\operatorname{dim}_{H} \mathcal{F}(\Phi)=\operatorname{dim}_{\mathrm{H}} \mathcal{F}\left(\Phi: n \rightarrow B^{n}\right) \quad \text { when } 1<B<\infty .
$$

Therefore, we can simply take the approximating function $\Phi(n):=B^{n}$ and rewrite the set $\mathcal{F}(\Phi)$ as

$$
\mathcal{F}(B)=\left\{x \in[0,1): \begin{array}{rl}
a_{n}(x) a_{n+1}(x) & \geq B^{n} \text { for infinitely many } n \in \mathbb{N} \text { and } \\
a_{n+1}(x) & <B^{n} \text { for all sufficiently large } n \in \mathbb{N}
\end{array}\right\} .
$$

The aim is to show $\operatorname{dim}_{H} \mathcal{F}(B)=t_{B}$. The details of the proof of Theorem 1.7 is divided into two further subsections. That is finding the upper bound

$$
\operatorname{dim}_{\mathrm{H}} \mathcal{F}(B) \leq t_{B}
$$

and the lower bound

$$
\operatorname{dim}_{\mathrm{H}} \mathcal{F}(B) \geq t_{B}
$$

separately. Taken together, this will conclude our proof for Case 1.

\subsubsection{The upper bound for $\mathcal{F}(B)$.}

For the upper bound of $\operatorname{dim}_{\mathrm{H}} \mathcal{F}(B)$, we split the set $\mathcal{F}(B)$ into two sets:

$$
\begin{aligned}
& \mathcal{F}_{1}(B)=\left\{x \in[0,1): a_{n}(x) \geq B^{n} \text { for infinitely many } n \in \mathbb{N}\right\} \text { and } \\
& \mathcal{F}_{2}(B)=\left\{x \in[0,1): \begin{array}{l}
1 \leq a_{n}(x) \leq B^{n}, a_{n+1}(x) \geq B^{n} / a_{n}(x) \text { for infinitely many } n \in \mathbb{N} \text { and } \\
a_{n+1}(x)<B^{n} \text { for all sufficiently large } n \in \mathbb{N}
\end{array}\right\} .
\end{aligned}
$$

From the definition of Hausdorff dimension it follows that

$$
\operatorname{dim}_{\mathrm{H}} \mathcal{F}(B)=\max \left\{\operatorname{dim}_{\mathrm{H}} \mathcal{F}_{1}(B), \operatorname{dim}_{\mathrm{H}} \mathcal{F}_{2}(B)\right\} .
$$

The Hausdorff dimension of $\mathcal{F}_{1}(B)$ follows from Theorem 1.3. So it remains to obtain the upper bound for the Hausdorff dimension of $\mathcal{F}_{2}(B)$. Recall that the pressure function $P(T,$. is monotonic with respect to the potential which implies then $s_{B} \leq t_{B}$. So, once we can show $\operatorname{dim}_{\mathrm{H}} \mathcal{F}_{2}(B) \leq t_{B}$, the upper bound for the $\operatorname{dim}_{\mathrm{H}} \mathcal{F}(B)$ follows.

Fix $\epsilon>0$ and let $s=t_{B}+2 \epsilon$. We will show that $\operatorname{dim}_{\mathrm{H}} \mathcal{F}_{2}(B) \leq s$. Assume that $0<s<1$.

By the definition of $t_{B}$, one has for any $n$ large,

$$
\sum_{a_{1}, \cdots, a_{n-1} \in \mathbb{N}}\left(\frac{1}{B^{n s} q_{n-1}^{2}}\right)^{s} \leq \sum_{a_{1}, \cdots, a_{n-1} \in \mathbb{N}}\left(\frac{1}{B^{n\left(t_{B}+\epsilon\right)} q_{n-1}^{2}}\right)^{t_{B}+\epsilon} \cdot B^{-n \epsilon^{2}} \leq B^{-n \epsilon^{2}} .
$$


Recall that

$$
\begin{aligned}
& \mathcal{F}_{2}(B)=\left\{x \in[0,1): \begin{array}{l}
1 \leq a_{n}(x) \leq B^{n}, a_{n+1}(x) \geq B^{n} / a_{n}(x) \text { for infinitely many } n \in \mathbb{N} \text { and } \\
a_{n+1}(x)<B^{n} \text { for all sufficiently large } n \in \mathbb{N}
\end{array}\right\} \\
& \subset\left\{x \in[0,1): 1 \leq a_{n}(x) \leq B^{n},\left(B^{n} / a_{n}(x)\right) \leq a_{n+1}(x)<B^{n} \text { for infinitely many } \in \mathbb{N}\right\} \\
&=\bigcap_{N=1}^{\infty} \bigcup_{n \geq N}\left\{x \in[0,1): 1 \leq a_{n}(x) \leq B^{n},\left(B^{n} / a_{n}(x)\right) \leq a_{n+1}(x)<B^{n}\right\} \\
&=\bigcap_{N=1}^{\infty} \bigcup_{n \geq N} \mathcal{F}_{I} \cup \mathcal{F}_{I I}
\end{aligned}
$$

where

$$
\begin{aligned}
& \mathcal{F}_{I}=\left\{x \in[0,1): 1 \leq a_{n}(x)<\alpha^{n},\left(B^{n} / a_{n}(x)\right) \leq a_{n+1}(x)<B^{n}\right\} \\
& \mathcal{F}_{I I}=\left\{x \in[0,1): \alpha^{n} \leq a_{n}(x) \leq B^{n},\left(B^{n} / a_{n}(x)\right) \leq a_{n+1}(x)<B^{n}\right\}
\end{aligned}
$$

and $\alpha^{n}>1$.

Next we will separately find suitable covering for set $\mathcal{F}_{I}$ and $\mathcal{F}_{I I}$ whereas the union of the coverings for both these sets will serve as an appropriate covering for $\mathcal{F}_{2}(B)$. To proceed, first assume that for a real number say $\alpha>1$ we have $\alpha^{n}>1$ for large enough $n \in \mathbb{N}$. Also assume that for some $0<s<1$ we have $\alpha=B^{s}$.

The set $\mathcal{F}_{I}$ can be covered by collections of fundamental cylinders $J_{n}$ of order $n$ :

$$
\begin{aligned}
\mathcal{F}_{I} & \subset\left\{x \in[0,1): 1 \leq a_{n}(x) \leq \alpha^{n},\left(B^{n} / a_{n}(x)\right) \leq a_{n+1}(x)\right\} \\
& =\bigcup_{a_{1}, \cdots, a_{n-1} \in \mathbb{N}}\left\{x \in[0,1): a_{k}(x)=a_{k}, 1 \leq k \leq n-1,1 \leq a_{n}(x) \leq \alpha^{n},\left(B^{n} / a_{n}(x)\right) \leq a_{n+1}(x)\right\} \\
& =\bigcup_{\substack{a_{1}, \cdots, a_{n-1} \in \mathbb{N} \\
1 \leq a_{n}<\alpha^{n}}} \bigcup_{a_{n+1} \geq B^{n} / a_{n}} I_{n+1}\left(a_{1}, \cdots, a_{n+1}\right) \\
& =\bigcup_{\substack{a_{1}, \cdots, a_{n-1} \in \mathbb{N}, 1 \leq a_{n} \leq \alpha^{n}}} J_{n}\left(a_{1}, \cdots, a_{n}\right) .
\end{aligned}
$$

Note that since

$$
J_{n}\left(a_{1}, \cdots, a_{n}\right)=\bigcup_{a_{n+1} \geq B^{n} / a_{n}} I_{n+1}\left(a_{1}, \cdots, a_{n+1}\right),
$$

therefore we have

$$
\left|J_{n}\left(a_{1}, \cdots, a_{n}\right)\right| \asymp \frac{1}{B^{n} a_{n} q_{n-1}^{2}} .
$$

Cover the set $\mathcal{F}_{I I}$ by the collection of fundamental cylinders $J_{n-1}$ of order $n-1$ :

$$
\begin{aligned}
\mathcal{F}_{I I} & \subset\left\{x \in[0,1): a_{n}(x) \geq \alpha^{n}\right\} \\
& =\bigcup_{a_{1}, \cdots, a_{n-1} \in \mathbb{N}}\left\{x \in[0,1): a_{k}(x)=a_{k}, 1 \leq k \leq n-1, a_{n}(x) \geq \alpha^{n}\right\} \\
& =\bigcup_{a_{1}, \cdots, a_{n-1} \in \mathbb{N}} \bigcup_{a_{n} \geq \alpha^{n}} I_{n}\left(a_{1}, \cdots, a_{n}\right) \\
& =\bigcup_{a_{1}, \cdots, a_{n-1} \in \mathbb{N}} J_{n-1}\left(a_{1}, \cdots, a_{n-1}\right) .
\end{aligned}
$$

Since

$$
J_{n-1}\left(a_{1}, \cdots, a_{n-1}\right)=\bigcup_{a_{n} \geq \alpha^{n}} I_{n}\left(a_{1}, \cdots, a_{n}\right)
$$


therefore we have

$$
\left|J_{n-1}\left(a_{1}, \cdots, a_{n-1}\right)\right| \asymp \frac{1}{\alpha^{n} q_{n-1}^{2}} .
$$

Now we consider the $s$-volume of the cover of $\mathcal{F}_{I} \cup \mathcal{F}_{I I}$ :

$$
\begin{aligned}
& \sum_{a_{1}, \cdots, a_{n-1} \in \mathbb{N}} \sum_{1 \leq a_{n} \leq \alpha^{n}}\left(\frac{1}{B^{n} a_{n} q_{n-1}^{2}}\right)^{s}+\sum_{a_{1}, \cdots, a_{n-1} \in \mathbb{N}}\left(\frac{1}{\alpha^{n} q_{n-1}^{2}}\right)^{s} \\
\asymp & \left.\sum_{a_{1}, \cdots, a_{n-1} \in \mathbb{N}} \alpha^{n(1-s)}\left(\frac{1}{B^{n} q_{n-1}^{2}}\right)^{s}+\sum_{a_{1}, \cdots, a_{n-1} \in \mathbb{N}}\left(\frac{1}{\alpha^{n} q_{n-1}^{2}}\right)^{s} \quad \text { (integrating on } a_{n}\right) \\
= & \sum_{a_{1}, \cdots, a_{n-1} \in \mathbb{N}}\left[\left(\frac{1}{\alpha^{n} q_{n-1}^{2}}\right)^{s}+\left(\frac{1}{\alpha^{n} q_{n-1}^{2}}\right)^{s}\right] \quad\left(\text { by } \alpha=B^{s}\right) \\
\asymp & \sum_{a_{1}, \cdots, a_{n-1} \in \mathbb{N}}\left(\frac{1}{B^{n s} q_{n-1}^{2}}\right)^{s} .
\end{aligned}
$$

Therefore, from equation (4.2), we obtain

$$
\mathcal{F}_{2}(B) \subset \bigcap_{N=1}^{\infty} \bigcup_{n \geq N}\left\{\bigcup_{\substack{a_{1}, \ldots, a_{n-1} \in \mathbb{N} \\ 1 \leq a_{n} \leq \alpha^{n}}} J_{n}\left(a_{1}, \cdots, a_{n}\right) \bigcup \bigcup_{a_{1}, \cdots, a_{n-1} \in \mathbb{N}} J_{n-1}\left(a_{1}, \cdots, a_{n-1}\right)\right\} .
$$

Thus from equationa (4.3) and (4.1), we obtain $s$-dimensional Hausdorff measure of $\mathcal{F}_{2}(B)$ as

$$
\mathcal{H}^{s}\left(\mathcal{F}_{2}(B)\right) \leq \liminf _{N \rightarrow \infty} \sum_{n \geq N}^{\infty} \sum_{a_{1}, \cdots, a_{n-1} \in \mathbb{N}}\left(\frac{1}{B^{n s} q_{n-1}^{2}}\right)^{s} \leq \liminf _{N \rightarrow \infty} \sum_{n \geq N}^{\infty} \frac{1}{B^{n \epsilon^{2}}}=0 .
$$

This gives $\operatorname{dim}_{\mathrm{H}} \mathcal{F}_{2}(B) \leq s=t_{B}+2 \epsilon$. Since $\epsilon>0$ is arbitrary, we have $\operatorname{dim}_{\mathrm{H}} \mathcal{F}_{2}(B) \leq t_{B}$. Consequently,

$$
\operatorname{dim}_{\mathrm{H}} \mathcal{F}(B) \leq t_{B}
$$

\subsubsection{The lower bound for $\mathcal{F}(B)$.}

In this subsection we will determine the lower bound for $\operatorname{dim}_{\mathrm{H}} \mathcal{F}(B)$. Here the pressure function material will be utilised.

To prove $\operatorname{dim}_{\mathrm{H}} \mathcal{F}(B) \geq t_{B}$ it is sufficient to show that $\operatorname{dim}_{\mathrm{H}} \mathcal{F}(B) \geq t_{L, B}(M)$ for all large enough $M$ and $L$ (Corollary 3.5). For this we will construct a subset $\mathcal{F}_{M}(B) \subset \mathcal{F}(B)$ and use the lower bound for Hausdorff dimension of $\mathcal{F}_{M}(B)$ to approximate that of $\mathcal{F}(B)$.

Fix $s<t_{L, B}(M)$. Let $\alpha=B^{s}$ where $\alpha \leq B$ and $\alpha^{n}>1$ for all large enough $n$. Choose a rapidly increasing sequence of integers $\left\{n_{k}\right\}_{k \geq 1}$ such that $n_{k} \gg n_{k-1}, \forall k$ and let $n_{0}=0$.

Define the subset $\mathcal{F}_{M}(B)$ of $\mathcal{F}(B)$ as follows

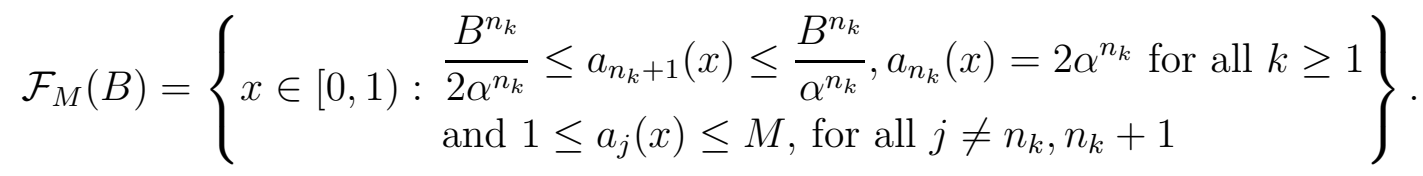


4.1.3. Structure of $\mathcal{F}_{M}(B)$.

For any $n \geq 1$, define the set of strings

$$
D_{n}=\left\{\left(a_{1}, \ldots, a_{n}\right) \in \mathbb{N}^{n}: \begin{array}{l}
\frac{B^{n_{k}}}{2 \alpha^{n_{k}}} \leq a_{n_{k}+1}(x) \leq \frac{B^{n_{k}}}{\alpha^{n_{k}}}, a_{n_{k}}(x)=2 \alpha^{n_{k}} \\
\text { and } 1 \leq a_{j}(x) \leq M, j \neq n_{k}, n_{k}+1
\end{array}\right\} .
$$

Recall that for any $n \geq 1$ and $\left(a_{1}, \ldots, a_{n}\right) \in D_{n}$, we call $I_{n}\left(a_{1}, \ldots, a_{n}\right)$ a basic cylinder of order $n$ and

$$
J_{n}:=J_{n}\left(a_{1}, \ldots, a_{n}\right):=\bigcup_{a_{n+1}} I_{n+1}\left(a_{1}, \ldots, a_{n}, a_{n+1}\right)
$$

a fundamental cylinder of order $n$, where the union in (4.6) is taken over all $a_{n+1}$ such that $\left(a_{1}, \ldots, a_{n}, a_{n+1}\right) \in D_{n+1}$.

Note that in (4.5) according to the limitations on the partial quotients we have three distinct cases for $J_{n}$. For $\left(a_{1}, \ldots, a_{n}, a_{n+1}\right) \in D_{n+1}$ :

$$
\begin{aligned}
n_{k-1}+1 & \leq n \leq n_{k}-2, & J_{n} & =\bigcup_{1 \leq a_{n+1} \leq M} I_{n+1}\left(a_{1}, \ldots, a_{n}, a_{n+1}\right), \\
n & =n_{k}-1, & J_{n} & =\bigcup_{a_{n+1}=2 \alpha^{n}} I_{n+1}\left(a_{1}, \ldots, a_{n}, a_{n_{1}+1}\right), \\
n & =n_{k}, & J_{n} & =\bigcup_{\frac{B^{n}}{2 \alpha^{n}} \leq a_{n+1} \leq \frac{B^{n}}{\alpha^{n}}} I_{n+1}\left(a_{1}, \ldots, a_{n}, a_{n+1}\right) .
\end{aligned}
$$

Then,

$$
\mathcal{F}_{M}(B)=\bigcap_{n=1}^{\infty} \bigcup_{\left(a_{1}, \ldots, a_{n}\right) \in D_{n}} J_{n}\left(a_{1}, \ldots, a_{n}\right)
$$

\subsubsection{Lengths of fundamental cylinders.}

In the following subsection we will estimate the lengths of the fundamental cylinders defined in subsection 4.1.3.

I. If $n_{k-1}+1 \leq n \leq n_{k}-2$ then from equation 4.7 and using equation 2.4 we have

$$
\begin{aligned}
\left|J_{n}\left(a_{1}, \ldots, a_{n}\right)\right| & =\sum_{1 \leq a_{n+1} \leq M}\left|I_{n+1}\left(a_{1}, \ldots, a_{n}, a_{n+1}\right)\right| \\
& =\sum_{1 \leq a_{n+1} \leq M} \frac{1}{q_{n+1}\left(q_{n+1}+q_{n}\right)} \\
& =\sum_{a_{n+1}=1}^{M} \frac{1}{q_{n}}\left(\frac{1}{q_{n+1}}-\frac{1}{q_{n+1}+q_{n}}\right) \\
& =\frac{1}{q_{n}} \sum_{a_{n+1}=1}^{M}\left(\frac{1}{a_{n+1} q_{n}+q_{n-1}}-\frac{1}{\left(a_{n+1}+1\right) q_{n}+q_{n-1}}\right) \\
& =\frac{1}{q_{n}}\left(\frac{1}{q_{n}+q_{n-1}}-\frac{1}{(M+1) q_{n}+q_{n-1}}\right) \\
& =\frac{M}{\left((M+1) q_{n}+q_{n-1}\right)\left(q_{n}+q_{n-1}\right)} .
\end{aligned}
$$

Also, from equation 4.10 we have

$$
\frac{1}{6 q_{n}^{2}} \leq\left|J_{n}\left(a_{1}, \cdots, a_{n}\right)\right| \leq \frac{1}{q_{n}^{2}}
$$


In particular for $n=n_{k}+1$,

$$
\frac{1}{24 B^{2 n} q_{n-2}^{2}} \leq\left|J_{n}\left(a_{1}, \cdots, a_{n}\right)\right| \leq \frac{1}{4 B^{2 n} q_{n-2}^{2}} .
$$

II. If $n=n_{k}-1$ then from equation 4.8 and following the same steps as for case $\mathbf{I}$ we have

$$
\left|J_{n}\left(a_{1}, \ldots, a_{n}\right)\right|=\frac{1}{\left(2 \alpha^{n} q_{n}+q_{n-1}\right)\left(\left(2 \alpha^{n}+1\right) q_{n}+q_{n-1}\right)}
$$

and

$$
\frac{1}{12 \alpha^{n+1} q_{n}^{2}} \leq\left|J_{n}\left(a_{1}, \cdots, a_{n}\right)\right| \leq \frac{1}{2 \alpha^{n+1} q_{n}^{2}}
$$

III. If $n=n_{k}$ then from equation 4.9 and following the similar steps as for $\mathbf{I}$ we obtain

$$
\left|J_{n}\left(a_{1}, \ldots, a_{n}\right)\right|=\frac{\frac{B^{n}}{2 \alpha^{n}}+1}{\left(\frac{B^{n}}{2 \alpha^{n}} q_{n}+q_{n-1}\right)\left(\left(\frac{B^{n}}{\alpha^{n}}+1\right) q_{n}+q_{n-1}\right)}
$$

and

$$
\frac{\alpha^{n}}{6 B^{n} q_{n}^{2}} \leq\left|J_{n}\left(a_{1}, \cdots, a_{n}\right)\right| \leq \frac{2 \alpha^{n}}{B^{n} q_{n}^{2}}
$$

Further,

$$
\frac{1}{32 \alpha^{n} B^{n} q_{n-1}^{2}} \leq\left|J_{n}\left(a_{1}, \cdots, a_{n}\right)\right| \leq \frac{1}{2 \alpha^{n} B^{n} q_{n-1}^{2}}
$$

\subsubsection{Supporting measure on $\mathcal{F}_{M}(B)$.}

To construct a suitable measure supported on $\mathcal{F}_{M}(B)$ first recall that $t_{L, B}(M)$ is the solution to

$$
\sum_{a_{1}, \ldots, a_{L} \in \mathcal{A}_{M}}\left(\frac{1}{B^{L s} q_{L}^{2}}\right)^{s}=1
$$

For $\alpha=B^{s}$ this sum becomes

$$
\sum_{a_{1}, \ldots, a_{L} \in \mathcal{A}_{M}}\left(\frac{1}{\alpha^{L} q_{L}^{2}}\right)^{s}=1
$$

Let $m_{k} L=n_{k}-n_{k-1}-1$ for any $k \geq 1$. Note that $m_{1} L=n_{1}-1$ since we have assumed $n_{0}=0$ and define

$$
w=\sum_{a_{1}, \ldots, a_{L} \in \mathcal{A}_{M}}\left(\frac{1}{\alpha^{L} q_{L}^{2}\left(a_{n_{k-1}+t+1}, \cdots, a_{n_{k-1}+(t+1) L}\right)}\right)^{s}
$$

where $0 \leq t \leq m_{k}-1$.

Step I. Let $1 \leq m \leq m_{1}$. We first define a positive measure for the fundamental cylinder $J_{m L}\left(a_{1}, \ldots, a_{m L}\right)$ as

$$
\mu\left(J_{m L}\left(a_{1}, \ldots, a_{m L}\right)\right)=\prod_{t=0}^{m-1} \frac{1}{w}\left(\frac{1}{\alpha^{L} q_{L}^{2}\left(a_{t L+1}, \ldots, a_{(t+1) L}\right)}\right)^{s},
$$

and then we distribute this measure uniformly over its next offspring.

Step II. When $n=m_{1} L=n_{1}-1$ then define a measure

$$
\mu\left(J_{m_{1} L}\left(a_{1}, \ldots, a_{m_{1} L}\right)\right)=\prod_{t=0}^{m_{1}-1} \frac{1}{w}\left(\frac{1}{\alpha^{L} q_{L}^{2}\left(a_{t L+1}, \ldots, a_{(t+1) L}\right)}\right)^{s} .
$$


Step III. When $n=m_{1} L+1=n_{1}$ then for $J_{n_{1}}\left(a_{1}, \ldots, a_{n_{1}}\right)$, define a measure

$$
\mu\left(J_{n_{1}}\left(a_{1}, \ldots, a_{n_{1}}\right)\right)=\frac{1}{2 \alpha^{n_{1}}} \mu\left(J_{n_{1}-1}\left(a_{1}, \ldots, a_{n_{1}-1}\right)\right)
$$

In other words, the measure of $J_{n_{1}-1}$ is uniformly distributed on its next offspring $J_{n_{1}}$.

Step IV. When $n=n_{1}+1$.

$$
\mu\left(J_{n_{1}+1}\left(a_{1}, \ldots, a_{n_{1}+1}\right)\right)=\frac{2 \alpha^{n_{1}}}{B^{n_{1}}} \mu\left(J_{n_{1}}\left(a_{1}, \ldots, a_{n_{1}}\right)\right)
$$

The measure of other fundamental cylinders of level less than $n_{1}-1$ is given by the consistency of a measure. To be more precise, for any $n<n_{1}-1$, suppose

$$
\mu\left(J_{n}\left(a_{1}, \cdots, a_{n}\right)\right)=\sum_{J_{m_{1} L} \subset J_{n}} \mu\left(J_{m_{1} L}\right) .
$$

So for any $m<m_{1}$, the measure of fundamental cylinder $J_{m L}$ is given by

$$
\mu\left(J_{m L}\left(a_{n_{k-1}+t+1} \cdots a_{n_{k-1}+(t+1) L}\right)\right)=\sum_{J_{m_{1} L} \subset J_{m L}} \mu\left(J_{m_{1}} L\right)=\prod_{t=0}^{m-1} \frac{1}{w}\left(\frac{1}{\alpha^{L} q_{L}^{2}\left(a_{t L+1}, \ldots, a_{(t+1) L}\right)}\right)^{s} .
$$

The measure of fundamental cylinders for other levels can be defined inductively.

For $k \geq 2$ define,

$$
\begin{gathered}
\mu\left(J_{n_{k}-1}\left(a_{1}, \ldots, a_{n_{k}-1}\right)\right)=\mu\left(J_{n_{k-1}+1}\left(a_{1}, \ldots, a_{n_{k-1}+1}\right)\right) \cdot \prod_{t=0}^{m_{k}-1} \frac{1}{w}\left(\frac{1}{\alpha^{L} q_{L}^{2}\left(a_{n_{k-1}+t L+1}, \ldots, a_{n_{k-1}+(t+1) L}\right)}\right)^{s}, \\
\mu\left(J_{n_{k}}\left(a_{1}, \ldots, a_{n_{k}}\right)\right)=\frac{1}{2 \alpha^{n_{k}}} \mu\left(J_{n_{k-1}}\left(a_{1}, \ldots, a_{n_{k-1}}\right)\right)
\end{gathered}
$$

and

$$
\mu\left(J_{n_{k}+1}\left(a_{1}, \ldots, a_{n_{k}+1}\right)\right)=\frac{2 \alpha^{n_{k}}}{B^{n_{k}}} \mu\left(J_{n_{k}}\left(a_{1}, \ldots, a_{n_{k}}\right)\right) .
$$

\subsubsection{The Hölder exponent of the measure $\mu$.}

Estimation of $\mu\left(J_{n}\left(a_{1}, \ldots, a_{n}\right)\right)$.

In this subsection we will estimate the measure $\mu$ of the fundamental cylinders defined above. For this we split the process into several cases. Recall that $\alpha^{n}>1$ for large enough $n$ which implies $\alpha^{L}>1$. For sufficiently large $k_{0}$ choose $\epsilon_{0}>\frac{n_{k-1}}{n_{k}}+\frac{1}{n_{k}}$ such that

$$
\frac{m_{k} L}{n_{k}}=\frac{n_{k}}{n_{k}}-\frac{n_{k-1}}{n_{k}}-\frac{1}{n_{k}} \geq 1-\epsilon_{0}, \text { for all } k>k_{0} .
$$

Case I. When $n=m L$ for some $1 \leq m<m_{1}$.

$$
\begin{aligned}
& \mu\left(J_{m L}\left(a_{1}, \ldots, a_{m L}\right)\right) \leq \prod_{t=0}^{m-1}\left(\frac{1}{\alpha^{L} q_{L}^{2}\left(a_{t L+1}, \ldots, a_{(t+1) L}\right)}\right)^{s} \leq \prod_{t=0}^{m-1}\left(\frac{1}{q_{L}^{2}\left(a_{t L+1}, \ldots, a_{(t+1) L}\right)}\right)^{s} . \\
& \mu\left(J_{m L}\left(a_{1}, \ldots, a_{m L}\right)\right) \leq\left(4^{m-1}\right)\left(\frac{1}{q_{m L}^{2}\left(a_{1}, \ldots, a_{m L}\right)}\right)^{s} \quad(\text { by Eq.2.6) } \\
& =\left(\frac{1}{q_{m L}^{2}\left(a_{1}, \ldots, a_{m L}\right)}\right)^{s-\frac{2}{L}} \quad\left(\text { by } \mathrm{P}_{1}\right) \\
& \leq 6\left|J_{m L}\left(a_{1}, \ldots, a_{m L}\right)\right|^{s-\frac{2}{L}} \quad(\text { by (4.11) }) \text {. }
\end{aligned}
$$


Case 2. When $n=m_{1} L=n_{1}-1$.

$$
\begin{aligned}
\mu\left(J_{m_{1} L}\left(a_{1}, \ldots, a_{m_{1} L}\right)\right) & \leq \prod_{t=0}^{m_{1}-1}\left(\frac{1}{\alpha^{L} q_{L}^{2}\left(a_{t L+1}, \ldots, a_{(t+1) L}\right)}\right)^{s} \\
& \leq\left(\frac{1}{\alpha^{m_{1} L}}\right)^{s}\left(\frac{1}{q_{m_{1} L}^{2}\left(a_{1}, \ldots, a_{m_{1} L}\right)}\right)^{s-\frac{2}{L}} \\
& \left.\leq\left(\frac{1}{\alpha^{1-\epsilon_{0}}}\right)^{s n_{1}}\left(\frac{1}{q_{m_{1} L}^{2}\left(a_{1}, \ldots, a_{m_{1} L}\right)}\right)^{s-\frac{2}{L}}(\text { by (4.15) })\right) \\
& \leq\left(\frac{1}{\alpha^{n_{1}} q_{n_{1}-1}^{2}}\right)^{s-\frac{2}{L}-\epsilon_{0}} \\
& \leq 12\left|J_{m_{1} L}\left(a_{1}, \ldots, a_{m_{1} L}\right)\right|^{s-\frac{2}{L}-\epsilon_{0}} \quad(\text { by (4.13) }) .
\end{aligned}
$$

Case 3. When $n=m_{1} L+1=n_{1}$.

$$
\begin{aligned}
\mu\left(J_{n_{1}}\left(a_{1}, \ldots, a_{n_{1}}\right)\right) & =\frac{1}{2 \alpha^{n_{1}}} \mu\left(J_{n_{1}-1}\left(a_{1}, \ldots, a_{n_{1}-1}\right)\right) \\
& \left.\leq \frac{1}{2 \alpha^{n_{1}}}\left(\frac{1}{\alpha^{n_{1}} q_{n_{1}-1}^{2}}\right)^{s-\frac{2}{L}-\epsilon_{0}} \quad(\text { by (4.16) })\right) \\
& =\frac{1}{2 B^{s n_{1}}}\left(\frac{1}{\alpha^{n_{1}} q_{n_{1}-1}^{2}}\right)^{s-\frac{2}{L}-\epsilon_{0}} \quad\left(\alpha=B^{s}\right) \\
& \leq \frac{1}{2}\left(\frac{1}{B^{n_{1}} \alpha^{n_{1}} q_{n_{1}-1}^{2}}\right)^{s-\frac{2}{L}-\epsilon_{0}} \\
& \leq 16\left|J_{n_{1}}\left(a_{1}, \ldots, a_{n_{1}}\right)\right|^{s-\frac{2}{L}-\epsilon_{0}}(\text { by (4.14) }) .
\end{aligned}
$$

Case 4. When $n=n_{1}+1$.

$$
\begin{aligned}
\mu\left(J_{n_{1}+1}\left(a_{1}, \ldots, a_{n_{1}+1}\right)\right) & =\frac{2 \alpha^{n_{1}}}{B^{n_{1}}} \mu\left(J_{n_{1}}\right) \\
& \leq \frac{2 \alpha^{n_{1}}}{2 B^{n_{1}}}\left(\frac{1}{B^{n_{1}} \alpha^{n_{1}} q_{n_{1}-1}^{2}}\right)^{s-\frac{2}{L}-\epsilon_{0}} \\
& \leq\left(\frac{1}{B^{2 n_{1}} \alpha^{n_{1}} q_{n_{1}-1}^{2}}\right)^{s-\frac{2}{L}-\epsilon_{0}} \\
& \leq 24\left|J_{n_{1}+1}\left(a_{1}, \ldots, a_{n_{1}+1}\right)\right|^{s-\frac{2}{L}-\epsilon_{0}}(\text { by }(4.12)) .
\end{aligned}
$$

Here for the second inequality, we use $B / \alpha \geq(B / \alpha)^{s}$ which is always true for $\alpha \leq B$ and $s \leq 1$.

For a general fundamental cylinder, we only give the estimation on the measure of $J_{n_{k}-1}\left(a_{1}, \ldots, a_{n_{k}-1}\right)$. The estimation for other fundamental cylinders can be carried out similarly. Recall that

$$
\mu\left(J_{n_{k}-1}\left(a_{1}, \ldots, a_{n_{k}-1}\right)\right)=\mu\left(J_{n_{k-1}+1}\left(a_{1}, \ldots, a_{n_{k-1}+1}\right)\right) \cdot \prod_{t=0}^{m_{k}-1} \frac{1}{w}\left(\frac{1}{\alpha^{L} q_{L}^{2}\left(a_{n_{k-1}+t L+1}, \ldots, a_{n_{k-1}+(t+1) L}\right)}\right)^{s}
$$


This further implies,

$$
\begin{aligned}
\mu\left(J_{n_{k}-1}\left(a_{1}, \ldots, a_{n_{k}-1}\right)\right) \leq\left[\prod_{j=1}^{k-1}\left(\frac{1}{B^{n_{j}}} \prod_{t=0}^{m_{j}-1}\left(\frac{1}{\alpha^{L} q_{L}^{2}\left(a_{n_{j-1}+t L+1}, \ldots, a_{n_{j-1}+(t+1) L}\right)}\right)^{s}\right)\right] \\
\leq\left[\prod_{j=1}^{k-1}\left(\frac{1}{B^{n_{j}}} \prod_{t=0}^{m_{j}-1}\left(\frac{1}{\alpha^{L} q_{L}^{2}\left(a_{n_{j-1}+t L+1}, \ldots, a_{n_{j-1}+(t+1) L}\right)}\right)^{s}\right)\right] \\
\cdot \prod_{t=0}^{m_{k}-1}\left(\frac{1}{\alpha^{L} q_{L}^{2}\left(a_{n_{k-1}+t L+1}, \ldots, a_{n_{k-1}+(t+1) L}\right)}\right)
\end{aligned}
$$

By similar arguments as used in (Case 4) for the first product and (Case 2) for the second product, we obtain

$$
\begin{aligned}
\mu\left(J_{n_{k}-1}\left(a_{1}, \ldots, a_{n_{k}-1}\right)\right) & \leq \prod_{j=1}^{k-1}\left(\frac{1}{B^{2 n_{j}} q_{m_{j} L}^{2}\left(a_{n_{j-1}+t L+1}, \ldots, a_{n_{j-1}+(t+1) L}\right)}\right)^{s-\frac{2}{L}-\epsilon} \\
\cdot\left(\frac{1}{\alpha^{n_{k}} q_{m_{k} L}^{2}\left(a_{n_{k-1}+t L+1}, \ldots, a_{n_{k-1}+(t+1) L}\right)}\right)^{s-\frac{2}{L}-\epsilon_{0}} & \leq 4^{2 k}\left(\frac{1}{\alpha^{n_{k}} q_{n_{k}-1}^{2}}\right)^{s-\frac{2}{L}-\epsilon_{0}} \leq\left(\frac{1}{\alpha^{n_{k}} q_{n_{k}-1}^{2}}\right)^{s-\frac{2}{L}-\epsilon_{0}-\frac{4}{L}} \\
& \left.\leq 12\left|J_{n_{k}-1}\left(a_{1}, \ldots, a_{n_{k}-1}\right)\right|^{s-\frac{6}{L}-\epsilon_{0}} \quad \text { (by (4.13) }\right) .
\end{aligned}
$$

Consequently,

$$
\begin{aligned}
\mu\left(J_{n_{k}}\left(a_{1}, \ldots, a_{n_{k}}\right)\right) & =\frac{1}{2 \alpha^{n_{k}}} \mu\left(J_{n_{k-1}}\left(a_{1}, \ldots, a_{n_{k-1}}\right)\right) \\
& \leq \frac{1}{2\left(B^{s}\right)^{n_{k}}}\left(\frac{1}{\alpha^{n_{k}} q_{n_{k}-1}^{2}}\right)^{s-\frac{2}{L}-\epsilon_{0}-\frac{4}{L}} \\
& \leq \frac{1}{2}\left(\frac{1}{B^{n_{k}} \alpha^{n_{k}} q_{n_{k}-1}^{2}}\right)^{s-\frac{2}{L}-\epsilon_{0}-\frac{4}{L}} \\
& \left.\leq 16\left|J_{n_{k}}\left(a_{1}, \ldots, a_{n_{k}}\right)\right|^{s-\frac{6}{L}-\epsilon_{0}} \quad \text { (by (4.14) }\right) .
\end{aligned}
$$

In summary, we have shown that

$$
\mu\left(J_{n}\left(a_{1}, \ldots, a_{n}\right)\right) \ll\left|J_{n}\left(a_{1}, \ldots, a_{n}\right)\right|^{s-\frac{2}{L}-\epsilon_{0}-\frac{4}{L}},
$$

for any $n \geq 1$ and $\left(a_{1}, \ldots, a_{n}\right) \in D_{n}$.

\subsubsection{Estimation of $\mu(B(x, r))$.}

First we estimate the gaps between the adjoint fundamental cylinders (defined in (4.6)) of same order which will be useful for estimating $\mu(B(x, r))$.

Let us start by assuming $n$ is even (similar steps can be followed when $n$ is odd). Then for $\left(a_{1}, a_{2}, \ldots, a_{n}\right) \in D_{n}$, given a fundamental cylinder $J_{n}\left(a_{1}, a_{2}, \ldots, a_{n}\right)$, represent the distance between $J_{n}\left(a_{1}, a_{2}, \ldots, a_{n}\right)$ and its left (respectively right) adjoint fundamental cylinder say

$$
J_{n}^{\prime}=J_{n}^{\prime}\left(a_{1}, \cdots, a_{n-1}, a_{n}-1\right) \text { (if exists) }
$$


(respectively, $\left.J_{n}^{\prime \prime}=J_{n}^{\prime \prime}\left(a_{1}, \cdots, a_{n-1}, a_{n}+1\right)\right)$ of order $n$ by $g^{l}\left(a_{1}, \ldots, a_{n}\right)\left(\right.$ respectively, $\left.g^{r}\left(a_{1}, \ldots, a_{n}\right)\right)$. Let

$$
G_{n}\left(a_{1}, a_{2}, \ldots, a_{n}\right)=\min \left\{g^{r}\left(a_{1}, a_{2}, \ldots, a_{n}\right), g^{l}\left(a_{1}, a_{2}, \ldots, a_{n}\right)\right\} .
$$

Again we will consider three different cases according to the range of $n$ as in (4.7) - (4.8) for $\mathcal{F}_{M}(B)$ in order to estimate the lengths of gaps on both sides of fundamental cylinders $J_{n}\left(a_{1}, a_{2}, \ldots, a_{n}\right)$.

Gap I. When $n_{k-1}+1 \leq n \leq n_{k}-2$, for all $k \geq 1$.

There exists a basic cylinder of order $n$ contained in $I_{n-1}\left(a_{1}, a_{2}, \ldots, a_{n-1}\right)$ which lies on the left of $I_{n}\left(a_{1}, a_{2}, \ldots, a_{n}\right)$, also there exists a basic cylinder of order $n$ contained in $I_{n-1}\left(a_{1}, a_{2}, \ldots, a_{n-1}\right)$ which lies on the right of $I_{n}\left(a_{1}, a_{2}, \ldots, a_{n}\right)$. In this case, $\left(a_{1}, a_{2}, \ldots, a_{n}-1\right) \in D_{n},\left(a_{1}, a_{2}, \ldots, a_{n}+1\right) \in D_{n}$, whereas $g^{l}\left(a_{1}, a_{2}, \ldots, a_{n}\right)$ is just the distance between the right endpoint of $J_{n}^{\prime}\left(a_{1}, a_{2}, \ldots, a_{n}-1\right)$ and the left endpoint of $J_{n}\left(a_{1}, a_{2}, \ldots, a_{n}\right)$.

The the right endpoint of $J_{n}^{\prime}\left(a_{1}, a_{2}, \ldots, a_{n}-1\right)$ is the same as the left endpoint of $I_{n}\left(a_{1}, a_{2}, \ldots, a_{n}\right)$. Since $n$ is even, from equation 2.1 this has formula $\frac{p_{n}}{q_{n}}$.

Note that the left endpoint of $J_{n}\left(a_{1}, a_{2}, \ldots, a_{n}\right)$ lies on the extreme left of all the constituent cylinders $\left\{I_{n+1}\left(a_{1}, a_{2}, \ldots, a_{n-1}, a_{n}, a_{n+1}\right): 1 \leq a_{n+1} \leq M\right\}$. This tells us that $a_{n+1}=M$. Since $n+1$ is odd, again from equation 2.1 this has formula

$$
\frac{\left(M p_{n}+p_{n-1}\right)+p_{n}}{\left(M q_{n}+q_{n-1}\right)+p_{n}}=\frac{(M+1) p_{n}+p_{n-1}}{(M+1) q_{n}+q_{n-1}} .
$$

Therefore, we have

$$
\begin{aligned}
g^{l}\left(a_{1}, a_{2}, \ldots, a_{n}\right) & =\frac{(M+1) p_{n}+p_{n-1}}{(M+1) q_{n}+q_{n-1}}-\frac{p_{n}}{q_{n}} \\
& =\frac{p_{n-1} q_{n}-q_{n-1} p_{n}}{\left((M+1) q_{n}+q_{n-1}\right) q_{n}}=\frac{1}{\left((M+1) q_{n}+q_{n-1}\right) q_{n}} .
\end{aligned}
$$

Whereas in this case $g^{r}\left(a_{1}, a_{2}, \ldots, a_{n}\right)$ is just the distance between the right endpoint of $J_{n}\left(a_{1}, a_{2}, \ldots, a_{n}\right)$ and the left endpoint of $J_{n}^{\prime \prime}\left(a_{1}, a_{2}, \ldots, a_{n}+1\right)$.

The right endpoint of $J_{n}\left(a_{1}, a_{2}, \ldots, a_{n}\right)$ is the same as the right endpoint of $I_{n}\left(a_{1}, a_{2}, \ldots, a_{n}\right)$. Since $n$ is even, again using equation 2.1 this has formula

$$
\frac{p_{n}+p_{n-1}}{q_{n}+q_{n-1}} .
$$

Also, the left endpoint of $J_{n}^{\prime \prime}\left(a_{1}, a_{2}, \ldots, a_{n}+1\right)$ lies on the extreme left of all the constituent cylinders $\left\{I_{n+1}\left(a_{1}, a_{2}, \ldots, a_{n-1}, a_{n}+1, a_{n+1}\right): 1 \leq a_{n+1} \leq M\right\}$. This tells us that $a_{n+1}=M$. Since $n+1$ is odd, from $\left(\mathrm{P}_{3}\right)$ this is given by

$$
\frac{(M+1)\left[\left(a_{n}+1\right) p_{n-1}+p_{n-2}\right]+p_{n-1}}{(M+1)\left[\left(a_{n}+1\right) q_{n-1}+q_{n-2}\right]+q_{n-1}}=\frac{(M+1)\left(p_{n}+p_{n-1}\right)+p_{n-1}}{(M+1)\left(q_{n}+q_{n-1}\right)+q_{n-1}} .
$$

Therefore,

$$
\begin{aligned}
g^{r}\left(a_{1}, a_{2}, \ldots, a_{n}\right) & =\frac{(M+1)\left(p_{n}+p_{n-1}\right)+p_{n-1}}{(M+1)\left(q_{n}+q_{n-1}\right)+q_{n-1}}-\frac{p_{n}+p_{n-1}}{q_{n}+q_{n-1}} \\
& =\frac{1}{\left((M+1)\left(q_{n}+q_{n-1}\right)+q_{n-1}\right)\left(q_{n}+q_{n-1}\right)}
\end{aligned}
$$

Hence

$$
G_{n}\left(a_{1}, a_{2}, \ldots, a_{n}\right)=\frac{1}{\left((M+1)\left(q_{n}+q_{n-1}\right)+q_{n-1}\right)\left(q_{n}+q_{n-1}\right)} .
$$


Also, by comparing $G_{n}\left(a_{1}, \ldots, a_{n}\right)$ with $J_{n}\left(a_{1}, \ldots, a_{n}\right)$ we notice that

$$
G_{n}\left(a_{1}, \ldots, a_{n}\right) \geq \frac{1}{2 M}\left|J_{n}\left(a_{1}, \ldots, a_{n}\right)\right| .
$$

Gap II. When $n=n_{k}-1$, we have

In this case the left gap $g^{l}\left(a_{1}, a_{2}, \ldots, a_{n}\right)$ is larger than the distance between the left endpoint of $I_{n}\left(a_{1}, a_{2}, \ldots, a_{n-1}, a_{n}\right)$ and the left endpoint of $J_{n}\left(a_{1}, a_{2}, \ldots, a_{n-1}, a_{n}\right)$ whereas the right gap $g^{r}\left(a_{1}, a_{2}, \ldots, a_{n}\right)$ is larger than the distance between the right endpoint of $I_{n}\left(a_{1}, a_{2}, \ldots, a_{n-1}, a_{n}\right)$ and the right endpoint of $J_{n}\left(a_{1}, a_{2}, \ldots, a_{n-1}, a_{n}\right)$.

Thus proceeding in the similar way as in Gap I, we obtain

$$
\begin{aligned}
g^{l}\left(a_{1}, a_{2}, \ldots, a_{n}\right) & \geq \frac{\left(2 \alpha^{n}+1\right) p_{n}+p_{n-1}}{\left(2 \alpha^{n}+1\right) q_{n}+q_{n-1}}-\frac{p_{n}}{q_{n}} \\
& =\frac{1}{\left(\left(2 \alpha^{n}+1\right) q_{n}+q_{n-1}\right) q_{n}} .
\end{aligned}
$$

and the left gap is

$$
\begin{aligned}
g^{r}\left(a_{1}, a_{2}, \ldots, a_{n}\right) & \geq \frac{p_{n}+p_{n-1}}{q_{n}+q_{n-1}}-\frac{\left(2 \alpha^{n}+1\right) p_{n}+p_{n-1}}{\left(2 \alpha^{n}+1\right) q_{n}+q_{n-1}} \\
& =\frac{1}{\left(\left(2 \alpha^{n}+1\right) p_{n}+p_{n-1}\right)\left(q_{n}+q_{n-1}\right)}
\end{aligned}
$$

Therefore,

$$
g^{r}\left(a_{1}, a_{2}, \ldots, a_{n}\right) \geq \frac{2 \alpha^{n}}{\left(\left(2 \alpha^{n}+1\right) q_{n}+q_{n-1}\right)\left(q_{n}+q_{n-1}\right)} .
$$

Thus

$$
G_{n}\left(a_{1}, a_{2}, \ldots, a_{n}\right) \geq \frac{1}{\left(\left(2 \alpha^{n}+1\right) q_{n}+q_{n-1}\right)\left(q_{n}+q_{n-1}\right)} .
$$

Further, in this case we have

$$
G_{n}\left(a_{1}, \ldots, a_{n}\right) \geq \frac{1}{2}\left|J_{n}\left(a_{1}, \ldots, a_{n}\right)\right| .
$$

Gap III. When $n=n_{k}$. Following the similar arguments as in Gap II we conclude

$$
\begin{aligned}
g^{l}\left(a_{1}, a_{2}, \ldots, a_{n}\right) & \geq \frac{\left(\frac{B^{n}}{\alpha^{n}}+1\right) p_{n}+p_{n-1}}{\left(\frac{B^{n}}{\alpha^{n}}+1\right) q_{n}+q_{n-1}}-\frac{p_{n}}{q_{n}} \\
& =\frac{1}{\left(\left(\frac{B^{n}}{\alpha^{n}}+1\right) q_{n}+q_{n-1}\right) q_{n}},
\end{aligned}
$$

and the right gap can be estimated as

$$
\begin{aligned}
g^{r}\left(a_{1}, a_{2}, \ldots, a_{n}\right) & \geq \frac{p_{n}+p_{n-1}}{q_{n}+q_{n-1}}-\frac{\left(\frac{B^{n}}{2 \alpha^{n}}+1\right) p_{n}+p_{n-1}}{\left(\frac{B^{n}}{2 \alpha^{n}}+1\right) q_{n}+q_{n-1}} \\
& =\frac{\frac{B^{n}}{2 \alpha^{n}}}{\left(\left(\frac{B^{n}}{2 \alpha^{n}}+1\right) q_{n}+q_{n-1}\right)\left(q_{n}+q_{n-1}\right)} .
\end{aligned}
$$

Thus we have,

and

$$
G_{n}\left(a_{1}, a_{2}, \ldots, a_{n}\right) \geq \frac{1}{\left(\left(\frac{B^{n}}{\alpha^{n}}+1\right) q_{n}+q_{n-1}\right)\left(q_{n}+q_{n-1}\right)},
$$

$$
G_{n}\left(a_{1}, \ldots, a_{n}\right) \geq \frac{1}{4}\left|J_{n}\left(a_{1}, \ldots, a_{n}\right)\right| .
$$


4.1.8. The measure $\mu$ on general ball $B(x, r)$.

Now we are in a position to estimate the measure $\mu$ on general ball $B(x, r)$. Fix $x \in \mathcal{F}_{M}(B)$ and let $B(x, r)$ be a ball centred at $x$ with radius $r$ small enough. There exists a unique sequence $a_{1}, a_{2}, \cdots a_{n}$ such that $x \in J_{n}\left(a_{1}, \cdots, a_{n}\right)$ for each $n \geq 1$ and

$$
G_{n+1}\left(a_{1}, \ldots, a_{n+1}\right) \leq r<G_{n}\left(a_{1}, \ldots, a_{n}\right) .
$$

It is clear, by the definition of $G_{n}$ that $B(x, r)$ can intersect only one fundamental cylinder of order $n$ i.e $J_{n}\left(a_{1}, \ldots, a_{n}\right)$.

Case I. $n=n_{k}$. Since in this case

$$
\left|I_{n_{k}+1}\left(a_{1}, \ldots, a_{n_{k}+1}\right)\right|=\frac{1}{q_{n_{k}+1}\left(q_{n_{k}+1}+q_{n_{k}}\right)} \geq \frac{1}{6 a_{n_{k+1}}^{2} q_{n_{k}}^{2}} \geq \frac{\alpha^{2 n_{k}}}{6 B^{2 n_{k}} q_{n_{k}}^{2}},
$$

the number of fundamental cylinders of order $n_{k}+1$ contained in $J_{n_{k}}\left(a_{1}, \ldots, a_{n_{k}}\right)$ that the ball $B(x, r)$ intersects is at most

$$
2 r \frac{6 B^{2 n_{k}}}{\alpha^{2 n_{k}}} q_{n_{k}}^{2}+2 \leq 24 r \frac{B^{2 n_{k}}}{\alpha^{2 n_{k}}} q_{n_{k}}^{2} .
$$

Therefore,

$$
\begin{aligned}
\mu(B(x, r)) & \leq \min \left\{\mu\left(J_{n_{k}}\right), 24 r \frac{B^{2 n_{k}}}{\alpha^{2 n_{k}}} q_{n_{k}}^{2} \mu\left(J_{n_{k}+1}\right)\right\} \\
& \leq \mu\left(J_{n_{k}}\right) \min \left\{1,48 r \frac{B^{n_{k}}}{\alpha^{n_{k}}} q_{n_{k}}^{2}\right\} \\
& \leq c\left|J_{n_{k}}\right|^{s-\frac{6}{L}-\epsilon_{0}} \min \left\{1,48 r \frac{B^{n_{k}}}{\alpha^{n_{k}}} q_{n_{k}}^{2}\right\} \\
& \leq c\left(\frac{2 \alpha^{n_{k}}}{B^{n_{k}} q_{n_{k}}^{2}}\right)^{s-\frac{6}{L}-\epsilon_{0}}\left(48 r \frac{B^{n_{k}}}{\alpha^{n_{k}}} q_{n_{k}}^{2}\right)^{s-\frac{6}{L}-\epsilon_{0}} \\
& \leq c_{0} r^{s-\frac{6}{L}-\epsilon_{0}} .
\end{aligned}
$$

Here we use $\min \{a, b\} \leq a^{1-s} b^{s}$ for any $a, b>0$ and $0 \leq s \leq 1$.

Case II. $n=n_{k}-1$. In this case, since

$$
\left|I_{n_{k}}\left(a_{1}, \ldots, a_{n_{k}}\right)\right|=\frac{1}{q_{n_{k}}\left(q_{n_{k}}+q_{n_{k}-1}\right)} \geq \frac{1}{6 a_{n_{k}}^{2} q_{n_{k}-1}^{2}} \geq \frac{1}{24 \alpha^{2 n_{k}} q_{n_{k}-1}^{2}},
$$

the number of fundamental intervals of order $n_{k}$ contained in $J_{n_{k}-1}\left(a_{1}, \ldots, a_{n_{k}-1}\right)$ that the ball $B(x, r)$ intersects is at most

$$
48 r \alpha^{2 n_{k}} q_{n_{k}-1}^{2}+2 \leq 96 r \alpha^{2 n_{k}} q_{n_{k}-1}^{2}
$$

Therefore,

$$
\begin{aligned}
\mu(B(x, r)) & \leq \min \left\{\mu\left(J_{n_{k}-1}\right), 96 r \alpha^{2 n_{k}} q_{n_{k}-1}^{2} \mu\left(J_{n_{k}}\right)\right\} \\
& \leq \mu\left(J_{n_{k}-1}\right) \min \left\{1,48 r \alpha^{n_{k}} q_{n_{k}-1}^{2}\right\} \\
& \leq 12\left|J_{n_{k}-1}\right|^{s-\frac{6}{L}-\epsilon_{0}} \min \left\{1,48 r \alpha^{n_{k}} q_{n_{k}-1}^{2}\right\} \\
& \leq 12\left(\frac{1}{2 \alpha^{n_{k}} q_{n_{k}-1}^{2}}\right)^{s-\frac{6}{L}-\epsilon_{0}}\left(48 r \alpha^{n_{k}} q_{n_{k}-1}^{2}\right)^{s-\frac{6}{L}-\epsilon_{0}} \\
& \leq c_{0} r^{s-\frac{6}{L}-\epsilon_{0}} .
\end{aligned}
$$


Case III. $n_{k-1}+1 \leq n \leq n_{k}-2$. Since in this case $1 \leq a_{n}(x) \leq M$ and $\left|J_{n}\right| \asymp 1 / q_{n}^{2}$ thus we have

$$
\begin{aligned}
\mu(B(x, r)) & \leq \mu\left(J_{n}\right) \leq c\left|J_{n}\right|^{s-\frac{6}{L}-\epsilon_{0}} \\
& \leq c\left(\frac{1}{q_{n}^{2}}\right)^{s-\frac{6}{L}-\epsilon 0} \\
& \leq c 4 M^{2}\left(\frac{1}{q_{n+1}^{2}}\right)^{s-\frac{6}{L}-\epsilon_{0}} \\
& \leq c 24 M^{2}\left|J_{n+1}\right|^{s-\frac{6}{L}-\epsilon 0} \\
& \leq c 48 M^{3} G_{n+1}^{s-\frac{6}{L}-\epsilon} \\
& \leq c 48 M^{3} r^{s-\frac{6}{L}-\epsilon} .
\end{aligned}
$$

Conclusion for the Lower Bound: Thus combining all the above cases and applying the mass distribution principle we have shown that $\operatorname{dim}_{\mathrm{H}} \mathcal{F}_{M}(B) \geq s-\frac{6}{L}-\epsilon_{0}$. Now letting $L \rightarrow \infty$, $M \rightarrow \infty$, by the choice of $\epsilon_{0}$ for all large enough $k$ and since $s<t_{B}$ is arbitrary, we have $s-\frac{6}{L}-\epsilon_{0} \rightarrow t_{B}$.

Thus we have,

$$
\operatorname{dim}_{\mathrm{H}} \mathcal{F}(B) \geq \operatorname{dim}_{\mathrm{H}} \mathcal{F}_{M}(B) \geq t_{B} .
$$

Taken together results (4.4) and (4.20), completes the proof of the desired theorem for the case $1<B<\infty$.

Next we prove Theorem 1.7 for the case when $B=\infty$.

4.2. Case 2. When $B=\infty$.

One can easily note that

$$
a_{n}(x) a_{n+1}(x) \geq \Phi(n) \Longrightarrow a_{n}(x) \geq \Phi(n)^{\frac{1}{2}} \text { or } a_{n+1}(x) \geq \Phi(n)^{\frac{1}{2}} .
$$

Thus

$$
\mathcal{F}(\Phi) \subseteq \mathcal{E}_{2}(\Phi) \subset \mathcal{G}_{1}(\Phi) \cup \mathcal{G}_{2}(\Phi)
$$

where

$$
\mathcal{G}_{1}(\Phi):=\left\{x \in[0,1): a_{n}(x) \geq \Phi(n)^{1 / 2} \text { for infinitely many } n \in \mathbb{N}\right\}
$$

and

$$
\mathcal{G}_{2}(\Phi):=\left\{x \in[0,1): a_{n+1}(x) \geq \Phi(n)^{1 / 2} \text { for infinitely many } n \in \mathbb{N}\right\} .
$$

2a. If $b=1$. Then for any $\delta>0, \frac{\log \log \Phi(n)}{n} \leq \log (1+\delta)$ that is $\Phi(n) \leq e^{(1+\delta)^{n}}$ for infinitely many $n \in \mathbb{N}$. Since

$$
\left\{x \in[0,1): a_{n}(x) \geq e^{(1+\delta)^{n}} \text { for all sufficiently large } n \in \mathbb{N}\right\} \subset \mathcal{F}(\Phi) .
$$

Therefore, by using lemma (2.3)

$$
\operatorname{dim}_{\mathrm{H}} \mathcal{F}(\Phi) \geq \lim _{\delta \rightarrow 0} \frac{1}{1+1+\delta}=\frac{1}{2} .
$$

Note that as $B=\infty$, therefore for any $C>1, \Phi(n) \geq C^{n}$ for all sufficiently large $n \in \mathbb{N}$. Thus by (4.21)

$$
\mathcal{F}(\Phi) \subseteq \mathcal{E}_{2}(\Phi) \subset\left\{x \in[0,1): a_{n}(x) \geq C^{n} \text { for infinitely many } \mathrm{n} \in \mathbb{N}\right\} .
$$

By Proposition (1.4), Theorem (1.3)

$$
\operatorname{dim}_{\mathrm{H}} \mathcal{F}(\Phi) \leq \lim _{C \rightarrow \infty} s_{C}=\frac{1}{2} .
$$


2b. If $1<b<\infty$. For any $\delta>0, \frac{\log \log \Phi(n)}{n} \leq \log (b+\delta)$ that is $\Phi(n) \leq e^{(b+\delta)^{n}}$ for infinitely many $n \in \mathbb{N}$, whereas $\Phi(n) \geq e^{(b-\delta)^{n}}$ for all sufficiently large $n \in \bar{N}$. Since

$\left\{x \in[0,1): a_{n}(x) \geq e^{(1+\delta)^{n}}\right.$ for all sufficiently large $\left.n \in \mathbb{N}\right\} \subset \mathcal{F}(\Phi)$.

Therefore, by using lemma (2.3)

$$
\operatorname{dim}_{\mathrm{H}} \mathcal{F}(\Phi) \geq \lim _{\delta \rightarrow 0} \frac{1}{1+b+\delta}=\frac{1}{1+b} .
$$

Further note that from the definition of the set $\mathcal{G}_{i}(\Phi)$ it is clear that $\mathcal{F}(\Phi) \subseteq \mathcal{E}_{2}(\Phi) \subset\left\{x \in[0,1): a_{n}(x) \geq e^{\frac{1}{2(b-\delta)}(b-\delta)^{n}}\right.$ for infinitely many $\left.n \in \mathbb{N}\right\}$

By Lemma 2.3

$$
\operatorname{dim}_{\mathrm{H}} \mathcal{F}(\Phi) \leq \lim _{\delta \rightarrow 0} \frac{1}{1+b-\delta}=\frac{1}{1+b}
$$

2c If $b=\infty$. Then by using the same argument as for showing the upper bound in case $2 \mathrm{~b}$ we have for any $C>1, \Phi(n) \geq e^{C^{n}}$ for all sufficiently large $n \in \mathbb{N}$. Thus by (4.21)

$$
\mathcal{F}(\Phi) \subseteq \mathcal{E}_{2}(\Phi) \subset\left\{x \in[0,1): a_{n}(x) \geq e^{C^{n}} \text { for infinitely many } \mathrm{n} \in \mathbb{N}\right\} .
$$

By Proposition (1.4), Theorem (1.3)

$$
\operatorname{dim}_{\mathrm{H}} \mathcal{F}(\Phi) \leq \lim _{C \rightarrow \infty} \frac{1}{C+1}=0 .
$$

This completes the proof of Theorem 1.7

Finally, we remark that it is possible to generalise the set $\mathcal{F}(\Phi)$ to the more general set of the form, for any $m \geq 2$

$$
\mathcal{F}_{m}(\Phi)=\left\{x \in[0,1): \begin{array}{l}
\prod_{k=1}^{m} a_{n+k-1}(x) \geq \Phi(n) \text { for infinitely many } n \in \mathbb{N} \text { and } \\
\prod_{k=1}^{m-1} a_{n+k-1}(x)<\Phi(n) \text { for all sufficiently large } n \in \mathbb{N}
\end{array}\right\}
$$

By following the same method as we have used for the proof of Theorem 1.7, we can show that:

Theorem 4.1. Let $\Phi: \mathbb{N} \rightarrow(1, \infty)$ be any function with $\lim _{n \rightarrow \infty} \Phi(n)=\infty$. Define $B, b$ as in Theorem 1.7. Then

1. $\operatorname{dim}_{\mathrm{H}} \mathcal{F}_{m}(\Phi)=\inf \left\{s \geq 0: \mathrm{P}\left(T,-g_{m} \log B-s \log \left|T^{\prime}\right|\right) \leq 0\right\}$ when $1<B<\infty$, where $g_{1}=s, g_{m}=\frac{s g_{m-1}(s)}{1-s+g_{m-1}(s)}$ for $m \geq 2$;

2. $\operatorname{dim}_{\mathrm{H}} \mathcal{F}_{m}(\Phi)=1 /(1+b)$ when $B=\infty$.

\section{REFERENCES}

[1] A. Bakhtawar, P. Bos, and M. Hussain. The sets of Dirichlet non-improvable numbers vs well-approximable numbers. In press: Ergod. Th. \& Dynam. Sys. Pre-Print: arXiv:1806.00618, 2019.

[2] F. Bernstein. Über eine Anwendung der Mengenlehre auf ein aus der Theorie der säkularen Störungen herrührendes Problem. Math. Ann., 71(3):417-439, 1911.

[3] E. Borel. Sur un problème de probabilités relatif aux fractions continues. Math. Ann., 72(4):578-584, 1912.

[4] H. Davenport and W. M. Schmidt. Dirichlet's theorem on diophantine approximation. In Symposia Mathematica, Vol. IV (INDAM, Rome, 1968/69), pages 113-132. Academic Press, London, 1970.

[5] D. J. Feng, J. Wu, J.-C. Liang, and S. Tseng. Appendix to the paper by T. łuczak-a simple proof of the lower bound: "On the fractional dimension of sets of continued fractions". Mathematika, 44(1):54-55, 1997. 
[6] I. J. Good. The fractional dimensional theory of continued fractions. Proc. Cambridge Philos. Soc., 37:199228, 1941.

[7] P. Hanus, R. D. Mauldin, and M. Urbański. Thermodynamic formalism and multifractal analysis of conformal infinite iterated function systems. Acta Math. Hungar., 96(1-2):27-98, 2002.

[8] L. Huang and J. Wu. Uniformly non-improvable dirichlet set via continued fractions. Proc. Amer. Math. Soc. DOI: https://doi.org/10.1090/proc/14587, 2019.

[9] M. Hussain, D. Kleinbock, N. Wadleigh, and B.-W. Wang. Hausdorff measure of sets of Dirichlet nonimprovable numbers. Mathematika, 64(2):502-518, 2018.

[10] A. Y. Khinchin. Continued fractions. The University of Chicago Press, Chicago, Ill.-London, 1964.

[11] D. Kleinbock and N. Wadleigh. A zero-one law for improvements to Dirichlet's Theorem. Proc. Amer. Math. Soc., 146(5):1833-1844, 2018.

[12] B. Li, B.-W. Wang, J. Wu, and J. Xu. The shrinking target problem in the dynamical system of continued fractions. Proc. Lond. Math. Soc. (3), 108(1):159-186, 2014.

[13] T. Łuczak. On the fractional dimension of sets of continued fractions. Mathematika, 44(1):50-53, 1997.

[14] R. D. Mauldin and M. Urbański. Dimensions and measures in infinite iterated function systems. Proc. London Math. Soc. (3), 73(1):105-154, 1996.

[15] R. D. Mauldin and M. Urbański. Conformal iterated function systems with applications to the geometry of continued fractions. Trans. Amer. Math. Soc., 351(12):4995-5025, 1999.

[16] R. D. Mauldin and M. Urbański. Graph directed Markov systems: geometry and dynamics of limit sets. Cambridge University Press, Cambridge, 2003.

[17] M. Pollicott. Lectures on fractals and dimension theory. https://homepages.warwick.ac.uk/ ${ }^{\sim}$ masdbl/dimensiontotal.pdf, 2005.

[18] P. Walters. An introduction to ergodic theory, volume 79 of Graduate Texts in Mathematics. Springer-Verlag, New York-Berlin, 1982.

[19] B.-W. Wang and J. Wu. Hausdorff dimension of certain sets arising in continued fraction expansions. $A d v$. Math., 218(5):1319-1339, 2008.

Department of Mathematics and Statistics, La Trobe University, PO Box 199, Bendigo 3552 , Australia.

E-mail address: A Bakhtawar: A.Bakhtawar@latrobe.edu.au

E-mail address: P Bos: P.Bos@latrobe.edu.au

E-mail address: M Hussain: m.hussain@latrobe.edu.au 